\title{
Characterizing the nano-structure and defect structure of nano-scaled non- ferrous structural alloys
}

Iman Ghamarian $^{1, *}$, Peyman Samimi ${ }^{1,2}$, Yue Liu ${ }^{2}$, Behrang Poorganji ${ }^{3}$, Vijay K. Vasudevan ${ }^{3}$, Peter C. Collins ${ }^{1,2}$

${ }^{1}$ Formerly with the Department of Materials Science and Engineering and the Center for Advanced Research and Testing, University of North Texas, Denton, TX 76203, USA, and currently with the Department of Materials Science and Engineering, Iowa State University, Ames, IA, 50011, USA

${ }^{2}$ Center for Advanced Non-Ferrous Structural Alloys (CANFSA), An NSF I/UCRC between the University of North Texas, Denton, TX 76203, USA and The Colorado School of Mines, Golden, CO 80401, USA

${ }^{3}$ Department of Mechanical and Materials Engineering, University of Cincinnati, Cincinnati, OH 45221, USA

Address correspondence to imanghamarian@yahoo.com

Phone: +1 515-294-5127

Fax: +1 515-294-1214

\begin{abstract}
The presence and interaction of nanotwins, geometrically necessary dislocations, and grain boundaries play a key role in the mechanical properties of nanostructured crystalline materials. Therefore, it is vital to determine the orientation, width and distance of nanotwins, the angle and axis of grain boundary misorientations as well as the type and the distributions of dislocations in an automatic and statistically meaningful fashion in a relatively large area. In this paper, such details are provided using a transmission electron microscope-based orientation microscopy technique called ASTAR ${ }^{\mathrm{TM}} /$ precession electron diffraction. The remarkable spatial resolution of this technique $(\sim 2 \mathrm{~nm})$ enables highly detailed characterization of nanotwins, grain boundaries and the configuration of dislocations. This orientation microscopy technique provides the raw
\end{abstract}


data required for the determination of these parameters. The procedures to post-process the ASTARTM/PED datasets in order to obtain the important (and currently largely hidden) details of nanotwins as well as quantifications of dislocation density distributions are described in this study.

\section{KEYWORDS}

nanoscale orientation microscopy, nanotwin characterization, ASTAR ${ }^{\mathrm{TM}}$, precession electron diffraction, spatial distribution of geometrically necessary dislocations, severely deformed metallic materials

\section{Introduction}

It is widely accepted that during the interaction of microstructure and defects, size effects begin to dominate when the scale of the microstructural features are small or when the number of defects is large. Thus, for nanostructured crystalline materials the presence and interaction of nanotwins, geometrically necessary dislocations, and grain boundaries play a key role in the overall balance of mechanical properties. Nanostructured metallic materials often exhibit superior properties such as high yield strength and fatigue resistivity with an attending debit in tensile ductility [1]. It may be possible to improve the overall balance of properties by tailoring the microstructural features, along with composition. For example, the introduction of a large volume of nanotwins (which form in metals with low or intermediate stacking fault energy such as $\mathrm{Ni}$ and $\mathrm{Cu}[2]$ ) simultaneously improves often competing mechanical properties (e.g. yield strength and ductility) without negatively or considerably affecting other physical properties, e.g., electrical conductivity [3]. This is achieved by promoting a ductile failure mode via the reduction of the spacing between multiple twins to an average inter-twin distance of <15 $\mathrm{nm}$ [4]. 
In reality, there is a significant lack of understanding of the phenomenon that occurs when the size-scale of the microstructure is quite small relative to the defect size. Indeed, with respect to plasticity there can be seemingly confusing and contradictory trends. For example, it is shown that for a case where the distance $(d)$ between nanotwins is larger than $150 \mathrm{~nm}$, the Hall-Petch equation is valid and the hardness follows $d^{-1 / 2}$ dependence. However, for smaller distance values (e.g. $d<100 \mathrm{~nm}$ ), a $d^{-1}$ dependence was observed. This deviation has been rationalized based on the nanotwin-dislocation interactions [5]. Another study by Zhao and LeSar showed that for thin grains that comprise a thin film, the exponent of the Hall-Petch relationship (a) for yield strength was highly dependent upon both the diameter as well as thickness. For thin grains (i.e., $t<250$ $\mathrm{nm}$ ), the exponent $a$ is $\sim 0.27$, whereas for thick grains (i.e., $t>1500 \mathrm{~nm}$ ), $a$ is $\sim 0.51$ [6]. Generally, the exponent term of the Hall-Petch equation associated with metallic materials may vary from 0.2 to 1 [7]. The breakaway from an 'idealized' Hall-Petch relationship can be assigned to various scenarios (e.g., rapid diffusional creep [8], presence of flaws [9], etc.) which govern the deformation once the grain size is below a critical value [10]. It is also observed to deviate from ideal in multi-phase microstructures where the length scale of the phases has a HallPetch relationship [11]. Different plastic deformation mechanisms/models have been proposed to explain softening with decreasing grain sizes, which are contrary to the idealized Hall-Petch relationship $[12,13]$. These include models on: breakdowns of the dislocation pile-up against grain boundaries [14-16]; so-called 'core and mantle' models [17-19] in which the grain interior is considered as the core and the grain boundary region is considered as the mantle; and the creation of a larger mean free path for dislocation movement via rotation of nanograins during plastic deformation and coalesce along the shear direction[20-22]. 
Similarly, it has been noted that Hall-Petch effect shows two opposite trends depending upon the thickness of the nanotwins in submicron grained $f c c$ metals [23]. While a normal Hall-Petch strengthening relationship is observed for nanotwins exhibiting a width greater than $15 \mathrm{~nm}$, grain boundary softening is the controlling phenomenon for the smaller nanotwins leading to softening. The deviation from a normal Hall-Petch relationship can be attributed to the loss of nanotwin boundary coherency (and their subsequent conversion to nanograin boundaries) due to the interaction of twins with dislocations. Moreover, dislocations which run parallel to nanotwins can pass the twin boundaries readily while others are blocked strongly [24]. Also, it has been shown that by reducing the thickness of the twins present, a transition of the rate controlling mechanism from intra-twin to twin-boundary-mediated processes happens [25]. Thus, it can be concluded that in order to better design materials, it is vital to correlate processing with the nanostructure. In order to make such a correlation, it is necessary to measure the orientation, width and distance of nanotwins as well as the type and configuration of dislocations in a statistically meaningful fashion, which requires that analysis be conducted over a relatively large $\operatorname{area}\left(\sim 10-100 \mu \mathrm{m}^{2}\right)$.

Since the interfacial area (e.g., grain boundaries and twin boundaries) is considerable in nanoscaled materials, interfaces play a key role in deformation specifically in intragranular slip, twinning [26] and in the interaction of nanotwins with grain boundaries. For instance, in nanograined materials, grain boundary sliding leads to nanocrack nucleation in the stress field of grain boundary disclinations (1D rotational defects [27]) [28]. In the case of $b c c$ and $f c c$ crystal structures, the main parameters which affect the nucleation of a crack from a nanotwin arrested at a grain boundary are the nanotwin width, the external stress, and the angle between the nanotwin and the grain boundary plane [26]. In addition, the grain boundary misorientation 
determines whether a nanotwin is allowed geometrically to cross the grain boundary and pass through the neighbor grain [26]. Recently, a model for nanotwins induced by grain boundaries during deformation has been proposed [29]. In this model, the importance of dislocation configuration close to grain boundaries and triple junctions is highlighted. Similar to the importance of high fidelity nanotwin-dislocation characterization, it is essential to gain a deep understanding of the details of the nanotwin-grain boundary interactions. Such details could be provided via an orientation microscopy technique with a spatial resolution of only a few nanometers.

Electron backscattered diffraction (EBSD), as a frequently used technique in such orientation microscopy studies, has (at best) an ultimate spatial resolution of 20-30 nm for slightly deformed materials [30] and greater than $100 \mathrm{~nm}$ for severely deformed materials [31, 32]. The spatial resolution of the EBSD technique can be improved to $10 \mathrm{~nm}$ by reducing the accelerating voltage to $7.5 \mathrm{kV}$, but with attending reductions in signal-to-noise ratio and experimental time [33]. Importantly, the probability of success in conducting such experiments is highly dependent upon the type of phosphor screen and experimental set-ups. A more exciting potential breakthrough, that is the direct detection of electron backscatter diffraction patterns using a complementary metal-oxide-semiconductor (CMOS) sensor, provides a high spatial resolution in addition to an improved signal-to-noise ratio and sharper bands in the recorded diffraction patterns, and the probable attending improvements in angular resolution at beam energies below the values at which conventional indirect detectors operate [34]. The limitations of the EBSD technique for the characterization of nanotwin lamella (with the maximum width size of less than $100 \mathrm{~nm}$ ) is reported in different studies [35-37]. A novel proposed procedure for orientation microscopy studies of nanotwins is a combination of ion channeling imaging and EBSD. In this approach, 
the location of nanotwins are detected by ion channeling imaging method and their orientations are determined based on the post processing of EBSD results [38]. However, this method is not a universal technique, owing to the intrinsic limitations of EBSD in general, such as the degradation of Kikuchi patterns at large strains [39].

Transmission Kikuchi diffraction (TKD) [40], also referred to as transmission electron forward scatter diffraction (t-EFSD) or transmission EBSD (t-EBSD) [41], is a new SEM based orientation microscopy technique with a spatial resolution of $\sim 2 \mathrm{~nm}$. In this technique, Kikuchi diffraction patterns associated with an electron transparent foil are recorded using a conventional EBSD detector located beneath the foil. In comparison to the conventional EBSD technique, the smaller beam-foil interaction volume enables TKD technique to characterize nanostructured and severely deformed metallic materials [42-44] due to limiting the orientation spread to a small volume [45]. The higher average energy and considerably narrower energy spread markedly reduce the multiple scattering in transmission mode which results in higher quality Kikuchi diffraction patterns [46]. Notably, numerous unindexed points and indexing errors associated with the orientation datasets collected using the TKD technique [45] are due to the presence of multiple grains along the thickness of the foil, degradation of Kikuchi patterns due to severe plastic deformation, the presence of very fine grains [39, 41, 47], etc. Also, the unusual projection geometry of the transmitted Kikuchi patterns in this technique may reduce the angular resolution to around $1^{\circ}[45]$.

Transmission electron microscopy (TEM) methods have been used commonly to study the structure and defects of nanomaterials [48]. These methods are complex and are limited to the analysis of a few features of interest. Dingley [49] developed an automated orientation microscopy technique for TEM in which crystal orientations are determined based on series of 
recorded dark field images. Similarly, an automated Kossel-Kikuchi line based method was applied to probe nanotwins by TEM [50]. Unfortunately, these two methods are not applied easily to highly strained materials. Another automated TEM-based orientation microscopy technique was developed through recording and indexing Kikuchi and spot diffraction patterns [51]. This powerful method is limited to samples and imaging conditions in which the kinematical theory of electron diffraction is valid - a condition that is often, regrettably, not satisfied under most experimental conditions.

ASTAR $^{\mathrm{TM}} /$ precession electron diffraction (ASTAR ${ }^{\mathrm{TM}} / \mathrm{PED}$ ) $[47,52]$ is an emerging TEM-based orientation microscopy technique that promises to change the way that the fine scale features (e.g., grains with diameter smaller than $100 \mathrm{~nm}$ ) of crystalline materials are studied. The spatial resolution of this technique is $\sim 2 \mathrm{~nm}$. The precession of the electron beam effectively changes the dynamical electron diffraction to quasi-kinematical electron diffraction [53], and not only sharpens the recorded spot diffraction patterns but also reduces/eliminates Kikuchi lines and residual background from the pattern. Precessing the beam also considerably reduces double diffraction and significantly helps in correctly indexing diffraction patterns that are said to suffer from a " $180^{\circ}$ ambiguity" [54]. The influence of beam precession on improving the acquired diffraction patterns is shown in Fig. 1a-d. Diffraction patterns which belong to a single grain within a thin film of a commercially pure titanium alloy and oriented close to a major zone axis were captured without precessing the incident beam (Fig. 1a) and with precessing the direct beam (Fig. 1b). Similar approach was followed for a crystal oriented far from a major zone axis (Fig. 1c-d). A relatively poor angular resolution (which represents the accuracy of the determination of crystal orientation

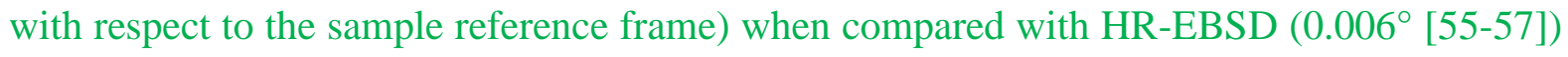


is one of the major limitations of ASTAR ${ }^{\mathrm{TM}} / \mathrm{PED}$ technique. The angular resolution of this technique improves by increasing the number of simulated diffraction pattern templates against which the experimentally acquired diffraction patterns are indexed. Templates are made by discretizing orientation space. By increasing the number of templates that are simulated, the accuracy of indexing is improved to some extent. During the template matching procedure, two Euler angles are determined via cross correlation of the diffraction pattern with all candidate templates. The third Euler angle is determined by rotating the most probable templates to find the highest overlap between templates and a diffraction pattern. This method of indexing the diffraction patterns results in anisotropic angular resolution. Additionally, the quality of the recorded diffraction patterns (including signal-to-noise ratio) impacts the accuracy of the image-template matching, as image processing steps (e.g., thresholding, noise reduction, etc.) are involved in the post-processing of the recorded diffraction patterns. Given that the intensity of the individual spots is a function of the structure factor, deviation parameter, and foil thickness, it is clear that the signal-to-noise ratio of any given spot, and hence its likelihood of being matched, is a function of the crystal orientation relative to both the beam direction and foil normal.

Further convolving the issue of angular resolution in highly-deformed materials is the fact that the lattice distortion is non-uniform. Not only does the elastic strain around individual dislocations depend upon (among other variables) the elastic stiffness tensor and dislocation line forces, but also configurations of multiple dislocations (and thus composite strains) depend upon the crystal orientation and spatial/crystallographic distributions of active slip systems (e.g., slip localization and preferential slip activity). 
These above caveats noted, it has been reported that the 'rule of thumb' average isotropic angular resolution of $\mathrm{ASTAR}^{\mathrm{TM}} / \mathrm{PED}$ technique is $\sim 0.8^{\circ}$ [47]. This has been shown to be improved by adopting an intensity-based approach to template matching. Specifically, it is possible to improve the angular resolution of ASTAR ${ }^{\mathrm{TM}} / \mathrm{PED}$ technique to $\sim 0.3^{\circ}$ [58]. Improvements in the angular resolution to $\sim 0.1^{\circ}$ should be sufficient to resolve single dislocations in TEM thin foils [59], and improvements in the spatial resolution coupled with improvements in angular resolution may allow individual dislocations or dislocation dipoles to be resolved.

As stated above, the accurate characterization of nanotwins requires that many attributes be analyzed, including the crystallography of nanotwins, the size of nanotwins and their proximity with other nanotwins, as well as their interactions with grain boundaries and dislocations. In this paper, ASTARTM/PED is introduced as a technique which provides the raw data needed to achieve the previously noted information. The procedures to post-process the ASTAR ${ }^{\mathrm{TM}} / \mathrm{PED}$ datasets in order to obtain the important (and currently largely hidden) details of nanotwins are described in the subsequent sections. The application of transmission-based orientation microscopy will not only be helpful in understanding plasticity in nanotwinned materials, but the other nanoscaled materials for which trends are seemingly contradictory. Notably, in this study, nanocrytsalline materials are considered as materials with the grain diameter within the range of $1 \mathrm{~nm}$ to $250 \mathrm{~nm}[60]$.

\section{Experimental methods}

\subsection{Experimental approaches}


An Inconel 617 sample with the approximate composition of Ni-22Cr-12Co-9Mo-1.2Al (all in weight percent) was rolled to a $60 \%$ reduction at room temperature and then annealed at 400 ${ }^{\circ} \mathrm{C}$ for one hour. A TEM sample was prepared along the rolling direction using conventional techniques involving dimpling and argon ion milling using a Fischine 1010 Ion Mill. Similarly, a TEM sample was prepared from a severely deformed commercially pure titanium alloy. TEMbased orientation microscopy was performed using ASTARTM/PED (NanoMEGAS, Brussels, Belgium) hardware and software package installed on an FEI Tecnai G2 F20 S-Twin FEG scanning/transmission electron microscope (S/TEM) operating at $200 \mathrm{KeV}$. It is imperative to note that various terminologies have been used for ASTARTM/PED technique. Some of these alternative terminologies are ACOM-TEM [61], IFPOM [62], D-STEM/PED [63], PACOM [47]. In this study, ASTAR ${ }^{\mathrm{TM}}$ represents the technology of rastering the direct beam over the entire region of interest and PED refers to the technology of precessing the direct beam from the optic axis.

The electron beam was aligned for spot size of $\sim 1 \mathrm{~nm}$ (which is equivalent to the spot size of nine on the FEI Tecnai [32]) and was precessed at an off-axis angle of $1.3^{\circ}$ with the precession frequency of $100 \mathrm{~Hz}$. The diffraction patterns were collected with the exposure time of $60 \mathrm{~ms}$. A $5^{*} 5 \mu \mathrm{m}^{2}$ region of each specimen scanned with the step size of $10 \mathrm{~nm}$. The diffraction patterns were acquired as $144 * 144$ pixel images at a camera length of $71 \mathrm{~mm}$ via an external high frame rate camera (a Stingray camera F-046, Allied Vision Technologies). Well-established image processing algorithms were applied to the recorded diffraction patterns to distinguish the diffraction spots from the background. Indexing the spot diffraction patterns was conducted by matching the recorded patterns, which were saved in a Blockfile, with the simulated diffraction patterns (> 5000 templates). The indexed pattern results were written as an $*$.ang file format for 
further post processing on TSL OIM ${ }^{\mathrm{TM}}$ software (TSL OIM ${ }^{\mathrm{TM}}$; EDAX, Mahwah, NJ, USA). The orientation dataset was cleaned up by disregarding data points with a confidence index smaller than 0.1. It is important to note that the confidence index, which is commonly used for EBSD technique, is analogous to reliability index for ASTARTM/PED technique [47]. Also, the reference frame of ASTAR ${ }^{\mathrm{TM}}$ was adjusted in accordance with the TSL OIM ${ }^{\mathrm{TM}}$ software reference frame [32].

\subsection{Analytical approaches}

The higher-order gradient method, an alternative to classical plasticity, is able to describe the behavior of crystalline materials with respect to their length scale [64]. Based on this method, it is possible to define plastic strain gradients in the framework of geometrically necessary dislocations (GNDs) in which the rotation gradients inside a Burgers circuit is assigned to the stored GND density. Preserving the lattice continuity requires the existence of GNDs (that is to say, the existence of GNDs is associated with lattice rotations). The lower bound of GND dislocation density can be calculated from an orientation dataset [65]. In this method, finite lattice rotations (i.e. the misorientation between an Euler angle set and its neighbors) determines the infinitesimal rotation components [66]. GND density calculations from an orientation dataset is explained nicely for EBSD [67-70] and ASTAR ${ }^{\mathrm{TM}} / \mathrm{PED}$ [59] acquired orientation datasets. For the sake of completeness, the procedure of GND density calculation from an orientation dataset is explained briefly.

Local lattice rotations (distortions) are calculated from the local disorientation values (the smallest misorientation between two Bunge Euler angle sets after applying crystal symmetries to the both orientation sets), according to Eq. 1, 


$$
\Delta \boldsymbol{w}=\frac{2 \cos ^{-1} \Delta q_{0}}{\sqrt{1-\Delta q_{0}^{2}}} \Delta \boldsymbol{q}
$$

Eq. 1

where $\Delta \boldsymbol{q}$ is the disorientation (in the quaternion representation) between the two orientation sets. The lattice curvature components can be calculated from the local lattice rotations (Eq. 2)

$$
\kappa_{k l}=\frac{\partial w_{k}}{\partial x_{l}}
$$

Eq. 2

where $k$ and $l$ are principal directions varying from 1 to 3 . Generally speaking, these methods provide $2 \mathrm{D}$ orientation datasets. As a result, there is no information regarding lattice curvatures in the third direction. This fact limits the total GND density calculations to the case of simple cubic crystal structure [71]. However, in the case of complex crystal structures (e.g., hcp), the GND density calculation method presented in this study approximates the lower bound values [72].

Of the two GND line energy minimization methods currently available [73, 74], the L1 method [66] was used to determine the configuration of dislocations which leads to the smallest dislocation (line) energy. In this method, the total line energy is defined as a function $(F)$ which is to be minimized. This function is the summation of the energy of all the possible pure edge and screw dislocation configurations which can exist in a crystal structure ${ }^{1}$. For instance, for the case of $f c c$ crystal structures, there are twelve pure edge and six pure screw dislocations [64] and for the case of ideal and arbitrary $h c p$ crystal structures, there are twenty four pure edge and nine pure screw dislocations $[67]^{2}$. The total line energy function, $F$, is minimized based on the simplex minimization algorithm [75] through applying three constraints as well as considering the fact that the energy of pure screw dislocations is proportional to $\boldsymbol{b}^{2}$ where $\boldsymbol{b}$ is the Burgers

\footnotetext{
${ }^{1}$ It is worth noting that mixed dislocations are recovered via an orthogonal decomposition of their pure edge and screw components.

${ }^{2}$ Regarding $h c p$ materials, different elements/materials will exhibit differences in the selection of which slip systems (i.e., basal, prismatic, pyramidal) are active.
} 
vector and $\left(\boldsymbol{b}^{2} /(1-v)\right)$ for pure edge dislocations where $v$ is the Poisson's ratio). The first constraint in the minimization approach is the fact that the dislocation density assigned to each dislocation type is either zero or positive. The second constraint is that the Burgers vector must be positive. In this case, for each individual dislocation type, two different directions for a Burgers vector are considered and finally the summation of their absolute values is reported as the dislocation density assigned to that specific dislocation type. Due to the second constraint, the total number of pure edge and screw dislocations in $f c c$ and $h c p$ crystal structures are considered as 36 [68] and 66 [67], respectively. The third constraint is governed by Eq. 3, in which the presence of the lattice curvatures is assigned to the existence of pure edge and screw dislocations. In a case in which the elastic strain gradient is negligible in comparison to the lattice rotation gradients, it is possible to determine five components of the dislocation density tensor and one difference between two other components from six lattice curvature components, as noted by Pantleon [65]. It has also been shown that Nye's analysis can be applied in the sample frame via solving Eq. 3 for a vector of dislocation densities ( $\rho$ ) using six lattice curvature components $\left(\frac{\partial \omega_{\mathrm{jk}}}{\partial \boldsymbol{x}_{\mathrm{i}}}\right)$ [76] where $b_{i}^{S}$ is the component of the sth Burgers vector along the $x_{i}$ th direction and $l_{i}^{S}$ is the component of the $s$ th line vector along the $x_{i}$ th direction [66]. 


$$
\left(\begin{array}{cc}
b_{1}^{l} l_{1}^{1}-0.5 \boldsymbol{b}^{1} \boldsymbol{l}^{l} & b_{1}^{s} l_{1}^{s}-0.5 \boldsymbol{b}^{s} \cdot \boldsymbol{l}^{s} \\
b_{1}^{l} l_{2}^{l} & b_{1}^{s} l_{2}^{s} \\
b_{1}^{l} l_{3}^{l} & b_{1}^{s} l_{3}^{s} \\
b_{2}^{l} l_{1}^{1} & b_{2}^{s} l_{1}^{s} \\
b_{2}^{l} l_{2}^{1}-0.5 \boldsymbol{b}^{1} \boldsymbol{l}^{l} & b_{2}^{s} l_{2}^{s}-0.5 \boldsymbol{b}^{s} \boldsymbol{l}^{s} \\
b_{2}^{l} l_{3}^{1} & b_{2}^{s} l_{3}^{s}
\end{array}\right)\left(\begin{array}{c}
\rho_{1} \\
\ldots \\
\rho_{s}
\end{array}\right)=\left(\begin{array}{c}
\frac{\partial \omega_{23}}{\partial \boldsymbol{x}_{1}} \\
\frac{\partial \omega_{31}}{\partial \boldsymbol{x}_{1}} \\
\frac{\partial \omega_{12}}{\partial \boldsymbol{x}_{1}} \\
\frac{\partial \omega_{23}}{\partial \boldsymbol{x}_{2}} \\
\frac{\partial \omega_{31}}{\partial \boldsymbol{x}_{2}} \\
\frac{\partial \omega_{12}}{\partial \boldsymbol{x}_{2}}
\end{array}\right)
$$

Eq. 3

Improvements in the angular resolution and calculations of the dislocation density distributions were conducted using a MATLAB code developed by the authors [59] and which has been integrated with the quantitative texture analysis software (MTEX 4.0 [77]) running on an eight core XEON, $2.4 \mathrm{GHz}$, Dell Precision T3600.

\section{Results and discussion}

Below, the characterization of nanotwins in a severely deformed nickel alloy (i.e., Inconel 617) is presented. In this section, given the novelty and newness of the approach, it is appropriate to present the methods of extraction of all the information required to study nanotwins from an orientation dataset collected using the ASTAR ${ }^{\mathrm{TM}} / \mathrm{PED}$ technique. The required information includes the crystallography of nanotwins, the size of nanotwins and their proximity with other nanotwins, the grain boundary angle and axis of misorientation as well as the spatial distribution of dislocation densities. Following this first section, the distribution of dislocation density and grain boundary analyses will be discussed for a severely deformed commercially pure titanium 
alloy which has a different crystal structure (i.e. $h c p$ ) than that of the nickel sample (i.e. $f c c$ ). Ultimately, it should be possible to fuse the different types of data - i.e., aspects of the microstructure and boundary character with the distribution of defects.

\subsection{Nanotwin characterization}

The bright field electron micrograph presented in Fig. 2a shows narrow and parallel features that exist in the severely deformed nickel sample. A higher magnification many-beam bright field micrograph (Fig. 2b) can be used to determine some important characteristics of these features (e.g., that their width is considerably less than $100 \mathrm{~nm}$ ). However, other important information, such as the nature of the feature (e.g., nanotwins or shear bands), is not clear. While conventional selected area diffraction (SAD) experiments could be used to determine whether such a feature is a nanotwin, SAD integrates all of the local crystal orientation information present within the confines of the virtual aperture, which in this case would capture multiple features of interest, thereby clouding the interpretations.

On the contrary, not only does the ASTAR ${ }^{\mathrm{TM}} / \mathrm{PED}$ technique (effectively, a convergent beam electron diffraction pattern) provide information from a much larger area and with a much finer spatial resolution, but the acquired orientation dataset can be post-processed to obtain additional information (e.g. local orientations, twin characteristics, grain boundary characteristics, dislocation density distribution). For example, an inverse pole figure (IPF) map of an

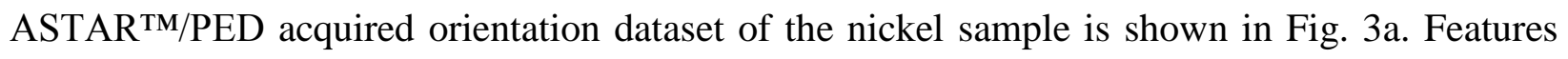
similar to those shown in Fig. 2(a,b) were probed using the ASTARTM/PED, and are observed here. The IPF map shows that the width of these bands varies from $\sim 50 \mathrm{~nm}$ to $\sim 400 \mathrm{~nm}$, and that the inter-twin distance ranges from $\sim 200 \mathrm{~nm}$ to $\sim 2000 \mathrm{~nm}$. Immediately, three observations are obvious, and none are surprising. Firstly, all are the same phase. Secondly, all of the detected and 
geometrically parallel bands share a similar crystallographic orientation, visualized by their assigned color. Lastly, their major axes are largely parallel. The latter two strongly suggest a certain crystallographic relationship. A portion of Fig. 3a which contains nano-size bands was enlarged and shown as Fig. 3b. The high spatial resolution of ASTAR ${ }^{\mathrm{TM}} / \mathrm{PED}$ enables the detection of bands with the average width of less than $30 \mathrm{~nm}$ (the band enclosed with $A$ and $B$ points in Fig. 3b). By post-processing the dataset using TSL OIM ${ }^{\mathrm{TM}}$ software, the green bands surrounded by dark lines have been identified as $\Sigma 3$ twins $\left(60^{\circ}<111>\right)$.

In order to confirm the existence of the nano-size twins that were detected by ASTAR $^{\mathrm{TM}} / \mathrm{PED}$, the original data belonging to a virtual line ( $A B$, see Fig. $\left.3 \mathrm{~b}\right)$ and the crystal orientations were further analyzed. The $A B$ line is comprised of eleven pixels. Each pixel represents one recorded diffraction pattern. Five of these diffraction patterns were located inside the twin area (daughter) and the rest belong to the matrix (parent). A diffraction pattern associated with a pixel located in the matrix is shown in Fig. 4a. The automated ASTAR ${ }^{\text {TM }}$ indexing software, i.e. ACOM (Automated Crystal Orientation Mapping), determined the simulated diffraction pattern shown in Fig. 4b exhibits the 'best matching' with respect to the recorded diffraction pattern. The schematic of the unit cell orientation relative to the electron beam direction (parallel to our viewing direction) which leads to the indexed diffraction pattern as well as the 111 pole figure are shown in Figs. 4(c,d), respectively. The corresponding information for a data point acquired from inside the nanotwin band is shown in Figs. 4(e-h). It is imperative to highlight the important role of ASTAR TM/PED in nanotwin characterization of severely deformed metallic materials by comparing Fig. 4a, Fig. 4e, Fig. 1b and Fig. 1d. Since Fig. 4a and Fig. 4e appear quite similar, the differences between the two images were calculated and are shown in Fig. 4k. 
The eight-bit recorded spot diffraction patterns depicted in Figs 4(a,e) were opened as intensity matrices in MATLAB software. Since the intensity of the real diffraction spots is larger than 75 (a.u.) in this study (based on the visual assessment), any intensity values smaller than 50 (a.u.) were changed to zero to remove the background (noise) of the images. Following this initial step, the difference matrix (i.e., $\left[\mathrm{M}_{\text {difference }}\right]=\left[\mathrm{M}_{\text {parent }}\right]-\left[\mathrm{M}_{\mathrm{twin}}\right]$ ) between two matrices (i.e., $\left[\mathrm{M}_{\text {parent }}\right]$ and $\left.\left[\mathrm{M}_{\mathrm{twin}}\right]\right)$ was determined.

The pixels of the diffraction patterns in which the intensity of the parent phase (Fig. 4a) is greater than daughter phase (i.e., $\left[\mathrm{M}_{\text {difference }}\right]>0$ ) are shown in light blue color in Fig. $4 \mathrm{k}$. Conversely, for pixels whose intensity is greater in the twin region (i.e., $\left[\mathrm{M}_{\text {difference }}\right]<0$ ) are visualized using a light green color in Fig. 4k. This figure demonstrates the fact that although some vital differences exist between the two diffraction patterns, it is very difficult to distinguish such differences without careful post-processing of the data. Finally, the point-to-point (local) and point-to-origin (global) misorientations along $A B$ line are shown in Fig. 4(1). The small variations in the local misorientation plot can be attributed to the $0.8^{\circ}$ angular resolution of ASTAR ${ }^{\mathrm{TM}} / \mathrm{PED}$ [47] (i.e. $0.8^{\circ}$ uncertainty in the orientation determination) and potentially the existence of defects (e.g. GNDs) which cause lattice distortions. This small variation in the local misorientation does not affect the $\Sigma 3$ twin $\left(60^{\circ}<111>\right)$ detection due to the fact that the misorientation made by the nanotwin is much larger than any small local misorientation variations due to noise ${ }^{3}$.

The importance of such techniques with a greatly improved spatial resolution, such as $\mathrm{ASTAR}^{\mathrm{TM}} / \mathrm{PED}$, can be illustrated by way of an example to determine the coherency of twin boundaries for twins $<100 \mathrm{~nm}$ thick. In order to consider the boundaries of $\Sigma 3$ twins $\left(60^{\circ}<111>\right)$

\footnotetext{
${ }^{3}$ Notably, the twin plane deviation tolerance was set to $2^{\circ}$ in the TSL OIM ${ }^{\mathrm{TM}}$ software.
} 
as coherent twin boundaries, the twin boundary plane must coincide with the twinning plane [78], i.e. the twin boundary plane must be parallel to the 111 planes on either sides of the twin. Since the acquired orientation dataset represents 2D information (no information is provided along the $\mathrm{z}$ axis of the studied sample), it is impossible to directly determine the 3D nature of the twin boundary with respect to the twining plane. However, according to the $3 \mathrm{D}$ orientation microscopy results provided by EBSD, it is shown that when the trace of the twin boundary is aligned with respect to the trace of a twin plane, the twin boundary is also aligned with the twinning plane in $90 \%$ of the cases [79]. For the sake of completeness, all the five boundary characters (i.e. three for the lattice misorientations and two for the orientation of boundary plane) can be derived either from 2D orientation dataset via following stereological method [80,81] or fusing the $2 \mathrm{D}$ orientation microscopy results acquired by ASTARTM/PED with the results of manual or automated tomographic tilt experiments, or top-bottom experiments. The 111 pole figure of the orientation dataset containing a nanotwin (Fig. 5b) is shown in Fig. 5a. The dashed circle in the pole figure includes two aligned poles which belong to the nanotwin and matrix. Since the twin boundary trace normal of the reconstructed twin boundary (i.e., the black vector in Fig. 5a and Fig. 5b) is well aligned with the poles surrounded by the circle, it can be concluded that the studied twin boundary is fully coherent.

\subsection{Dislocation density analyses}

Orientation microscopy of the severely deformed commercially pure titanium alloy was done by ASTARTM/PED. The quaternion misorientation plot (Fig. 6a) shows nano-size grains. Slightly more than a hundred grains, defined by minimum misorientation between two grains of $5^{\circ}$, were identified automatically by TSL OIM ${ }^{\mathrm{TM}}$ software in a $\sim 1.15 \mu \mathrm{m}^{2}$ area. Based on the color legend shown in Fig. 6b, it is clear that there is a significant orientation variation (and, consequently, an 
expected broad variation in mechanical properties) in most of the grains with respect to their neighbors. Such orientation information is crucial when characterizing severely deformed materials. The grain size distribution is shown in Fig. 6c. This plot quantitatively indicates that a considerable number fraction of the detected grains have a diameter smaller than $100 \mathrm{~nm}$ (i.e. nano-size grains). In addition, the grain boundary angle and axis of misorientation can be determined readily by matching the color and contrast with the color legend shown in Fig. 6b and finding the location of the matched color and contrast in the triangle, respectively. As an example, an overlay of grain boundaries expressed by their quaternion (as a color and contrast) with the index map is shown in Fig. 6(d). All the presented results in Fig. 6 (i.e. grain boundary misorientation angle and axis as well as the grain size distribution) will enable the development of polycrystal grain refinement models $[82,83]$ and the rigorous assessment of the effect of grain refinement on misorientation correlation between a grain and its neighbors $[84,85]$.

The dislocation density distribution maps of <a>-type pure screw dislocations with the Burgers vector of $0.3[\overline{2} 110]$ and $\langle\boldsymbol{c}+\boldsymbol{a}\rangle$-type pyramidal pure edge dislocations with the Burgers vector of $0.3[\overline{2} 11 \overline{3}]$ are shown in Fig. 7a and Fig. 7b, respectively. The minimum detectable dislocation density was calculated according to Eq. 4 [74],

$$
\rho_{\text {min }}^{G N D}=\frac{\text { Angular resolution }(\mathrm{rad})}{\text { Step size } * \text { Burger's vector length }}
$$

where the improved angular resolution via Kuwahara filter was $0.4^{\circ}$, the step size was $10 \mathrm{~nm}$ and the Burgers vector length was $0.295 \mathrm{~nm}$ for $\langle\boldsymbol{a}>$-type dislocations in an alpha phase titanium. Interestingly, both $\langle\boldsymbol{a}\rangle$-type and $\langle\boldsymbol{c}+\boldsymbol{a}\rangle$-type dislocation density distribution maps presented in this study are consistent with the TEM-conducted direct observations of these dislocation types in the severely deformed area of a nanoindentation located in the $\alpha$ phase of an $\alpha / \beta$ titanium alloy [86]. It is shown that the population of $\langle\boldsymbol{c}+\boldsymbol{a}\rangle$-type dislocations is more than the $\langle\boldsymbol{a}\rangle$-type dislocations in the area beneath the nanoindentation in which the material was deformed largely [86]. Importantly, the same observations are made here. 
Pivotal information can be derived from the dislocation density distribution plots [59]. For instance, the dislocation architecture around a nanotwin or a grain boundary can be studied simply via looking at this type of plots. Also, the effect of the orientation of a neighboring grain and its deformation on the dislocation density evolution of the first grain can be studied. As an example, two adjacent grains which are surrounded by a red rectangle in Fig. $7 \mathrm{~b}$ represent two opposite dislocation density evolution scenarios, i.e. one is almost devoid of dislocations while the other one has, essentially, a uniform dislocation density distribution. Notably, dislocation density distributions [87] and dislocation-grain boundary interactions [88] provide bases for plasticity studies [89]. The fusion of microstructure and defect structure data is seen as an essential component of integrated computational/experimental strategies that may provide answers, and thus allow the unresolved questions associated with plasticity to be resolved.

\section{Conclusions}

Given that the plasticity of structural metallic materials is ultimately controlled by the interaction of the defects (i.e., dislocations and deformation twins) with the microstructural features, it is imperative to be able to link defects and microstructure. This paper has taken two materials where the scale of the microstructural features is at the nano-scale, and thus which exhibit interesting mechanical properties. In order to simultaneously resolve the nanostructure and defect structures, the ASTARTM/PED technique is presented. This technique is a TEM-based orientation microscopy technique, and has been used to characterize the following attributes of the microstructure and the defect structure.

- For nanotwins, it is possible to accurately determine their width, orientation, their intertwine distance and their coherency.

- The distribution of dislocation density of geometrically necessary dislocations can be determined via using the orientation dataset acquired by this technique. Poor angular 
resolution of this technique is the main problem of resolving dislocation densities in materials that have not been subjected to severe plastic deformation.

- The angle and axis of misorientation of grain boundaries can be determined using this technique.

\section{Acknowledgements}

The authors gratefully acknowledge the University of North Texas; the Center for Advanced Non-Ferrous Structural Alloys, which is a joint industry-university center between the Colorado School of Mines and the University of North Texas (NSF award no. 1134873); the Center for Advanced Research and Testing at the University of North Texas. In addition, BP and VKV are grateful for financial support of this research by the Nuclear Energy University Program (NEUP) of the US Department of Energy contract \#88635 issued under prime contract DE-AC0705ID14517 to Battelle Energy Alliance, LLC. BP and VKV also gratefully acknowledge the contribution of the State of Ohio, Department of Development and Third Frontier Commission, which provided funding in support of "Ohio Center for Laser Shock Processing for Advanced Materials and Devices" at the University of Cincinnati and the equipment in the Center that was used in this work.

\section{References}

[1] M.A. Meyers, K.K. Chawla, Mechanical behavior of materials, Cambridge University Press Cambridge, 2009.

[2] K. Yu, D. Bufford, Y. Chen, Y. Liu, H. Wang, X. Zhang, Basic criteria for formation of growth twins in high stacking fault energy metals, Appl Phys Lett, 103 (2013) 181903.

[3] K. Lu, L. Lu, S. Suresh, Strengthening materials by engineering coherent internal boundaries at the nanoscale, Science, 324 (2009) 349-352.

[4] Y.-W. Zhang, Mechanical properties: Nanotwins only, Nat. Nanotechnol., 7 (2012) 551-552. [5] L.L. Shaw, A.L. Ortiz, J.C. Villegas, Hall-Petch relationship in a nanotwinned nickel alloy, Scripta Mater., 58 (2008) 951-954. 
[6] C. Zhou, R. LeSar, Dislocation dynamics simulations of plasticity in polycrystalline thin films, Int J Plast, 30-31 (2012) 185-201.

[7] D.J. Dunstan, A.J. Bushby, Grain size dependence of the strength of metals: The Hall-Petch effect does not scale as the inverse square root of grain size, Int J Plast, 53 (2014) 56-65.

[8] A. Chokshi, A. Rosen, J. Karch, H. Gleiter, On the validity of the Hall-Petch relationship in nanocrystalline materials, Scripta Metallurgica, 23 (1989) 1679-1683.

[9] J.R. Weertman, Hall-Petch strengthening in nanocrystalline metals, Mater. Sci. Eng. A, 166 (1993) 161-167.

[10] C.C. Koch, Optimization of strength and ductility in nanocrystalline and ultrafine grained metals, Scripta Mater., 49 (2003) 657-662.

[11] I. Ghamarian, P. Samimi, V. Dixit, P.C. Collins, A constitutive equation relating composition and microstructure to properties in Ti-6-4 - as derived using a novel integrated computational approach Metall Mater Trans A, (2015) doi:10.1007/s11661-11015-13072-11664. [12] H. Conrad, Grain-size dependence of the flow stress of $\mathrm{Cu}$ from millimeters to nanometers, Metall Mater Trans A, 35 (2004) 2681-2695.

[13] H. Conrad, J. Narayan, Mechanisms for grain size hardening and softening in Zn, Acta Mater., 50 (2002) 5067-5078.

[14] C. Pande, R. Masumura, R. Armstrong, Pile-up based Hall-Petch relation for nanoscale materials, Nanostructured materials, 2 (1993) 323-331.

[15] R. Armstrong, G. Hughes, The limiting strength properties of nanocrystalline materials, Univ. of Maryland, College Park, MD (US), 1999.

[16] T. Smith, R. Armstrong, P. Hazzledine, R. Masumura, C. Pande, M. Otooni, R. Armstrong, N. Grant, K. Ishizaki, Grain Size and Mechanical Properties-Fundamentals and Applications, Materials Research Society, Pittsburgh, (1995).

[17] J.P. Hirth, J. Lothe, Theory of dislocations, 1982.

[18] A. Thompson, Work hardening in tension and fatigue, AIME, (1977) 89-126.

[19] H. Margolin, Polycrystalline yielding_-perspectives on its onset, Acta Mater., 46 (1998) 6305-6309.

[20] D. Jia, Y. Wang, K. Ramesh, E. Ma, Y. Zhu, R. Valiev, Deformation behavior and plastic instabilities of ultrafine-grained titanium, Appl Phys Lett, 79 (2001) 611-613.

[21] Y. Wang, E. Ma, M. Chen, Enhanced tensile ductility and toughness in nanostructured $\mathrm{Cu}$, Appl Phys Lett, 80 (2002) 2395-2397.

[22] D. Jia, K.T. Ramesh, E. Ma, Failure mode and dynamic behavior of nanophase iron under compression, Scripta Mater., 42 (1999) 73-78.

[23] G. Malygin, Plasticity and strength of FCC metals with a nanotwinned submicrograined structure, Physics of the Solid State, 53 (2011) 763-767.

[24] D. Jang, X. Li, H. Gao, J.R. Greer, Deformation mechanisms in nanotwinned metal nanopillars, Nat. Nanotechnol., 7 (2012) 594-601.

[25] L. Lu, M. Dao, T. Zhu, J. Li, Size dependence of rate-controlling deformation mechanisms in nanotwinned copper, Scripta Mater., 60 (2009) 1062-1066.

[26] I. Ovid'ko, A. Sheinerman, Nanoscale cracks at deformation twins stopped by grain boundaries in bulk and thin-film materials with nanocrystalline and ultrafine-grained structures, J. Phys. D: Appl. Phys., 47 (2014) 015307.

[27] A.E. Romanov, A.L. Kolesnikova, Application of disclination concept to solid structures, Prog Mater Sci, 54 (2009) 740-769. 
[28] I.A. Ovid'ko, A.G. Sheinerman, Enhanced ductility of nanomaterials through optimization of grain boundary sliding and diffusion processes, Acta Mater., 57 (2009) 2217-2228.

[29] I.A. Ovid'ko, N.V. Skiba, Nanotwins induced by grain boundary deformation processes in nanomaterials, Scripta Mater., 71 (2014) 33-36.

[30] F. Humphreys, Y. Huang, I. Brough, C. Harris, Electron backscatter diffraction of grain and subgrain structures - resolution considerations, J. Microsc., 195 (1999) 212-216.

[31] S. Zaefferer, On the formation mechanisms, spatial resolution and intensity of backscatter Kikuchi patterns, Ultramicroscopy, 107 (2007) 254-266.

[32] X. Liu, N. Nuhfer, A. Rollett, S. Sinha, S.-B. Lee, J. Carpenter, J. LeDonne, A. Darbal, K.

Barmak, Interfacial orientation and misorientation relationships in nanolamellar $\mathrm{Cu} / \mathrm{Nb}$

composites using transmission-electron-microscope-based orientation and phase mapping, Acta

Mater., 64 (2013) 333-344.

[33] D. Steinmetz, S. Zaefferer, Towards ultrahigh resolution EBSD by low accelerating voltage, Mater Sci Technol, 26 (2010) 640-645.

[34] A.J. Wilkinson, G. Moldovan, T.B. Britton, A. Bewick, R. Clough, A.I. Kirkland, Direct detection of electron backscatter diffraction patterns, Phys. Rev. Lett., 111 (2013) 065506.

[35] S. Dancette, L. Delannay, K. Renard, M. Melchior, P. Jacques, Crystal plasticity modeling of texture development and hardening in TWIP steels, Acta Mater., 60 (2012) 2135-2145.

[36] D.R. Steinmetz, T. Jäpel, B. Wietbrock, P. Eisenlohr, I. Gutierrez-Urrutia, A. Saeed-Akbari, T. Hickel, F. Roters, D. Raabe, Revealing the strain-hardening behavior of twinning-induced plasticity steels: Theory, simulations, experiments, Acta Mater., 61 (2013) 494-510.

[37] D. Barbier, N. Gey, S. Allain, N. Bozzolo, M. Humbert, Analysis of the tensile behavior of a TWIP steel based on the texture and microstructure evolutions, Mater. Sci. Eng. A, 500 (2009) 196-206.

[38] H. Alimadadi, A. Fanta, K. Pantleon, The complementary use of electron backscatter diffraction and ion channelling imaging for the characterization of nanotwins, J. Microsc., 249 (2013) 111-118.

[39] S.I. Wright, M.M. Nowell, D.P. Field, A review of strain analysis using electron backscatter diffraction, Microsc. Microanal., 17 (2011) 316-329.

[40] P.W. Trimby, Orientation mapping of nanostructured materials using transmission Kikuchi diffraction in the scanning electron microscope, Ultramicroscopy, 120 (2012) 16-24.

[41] N. Brodusch, H. Demers, R. Gauvin, Nanometres-resolution Kikuchi patterns from materials science specimens with transmission electron forward scatter diffraction in the scanning electron microscope, J. Microsc., 250 (2013) 1-14.

[42] K. Tugcu, G. Sha, X.Z. Liao, P. Trimby, J.H. Xia, M.Y. Murashkin, Y. Xie, R.Z. Valiev, S.P. Ringer, Enhanced grain refinement of an $\mathrm{Al}-\mathrm{Mg}-\mathrm{Si}$ alloy by high-pressure torsion processing at $100{ }^{\circ} \mathrm{C}$, Mater. Sci. Eng. A, 552 (2012) 415-418.

[43] J.L. Sun, P.W. Trimby, F.K. Yan, X.Z. Liao, N.R. Tao, J.T. Wang, Grain size effect on deformation twinning propensity in ultrafine-grained hexagonal close-packed titanium, Scripta Mater., 69 (2013) 428-431.

[44] J. Kacher, P. Elizaga, S.D. House, K. Hattar, M. Nowell, I.M. Robertson, Thermal stability of Ni/NiO multilayers, Mater. Sci. Eng. A, 568 (2013) 49-60.

[45] P.W. Trimby, Y. Cao, Z. Chen, S. Han, K.J. Hemker, J. Lian, X. Liao, P. Rottmann, S.

Samudrala, J. Sun, J.T. Wang, J. Wheeler, J.M. Cairney, Characterizing deformed ultrafinegrained and nanocrystalline materials using transmission Kikuchi diffraction in a scanning electron microscope, Acta Mater., 62 (2014) 69-80. 
[46] R. Keller, R. Geiss, Transmission EBSD from $10 \mathrm{~nm}$ domains in a scanning electron microscope, J. Microsc., 245 (2012) 245-251.

[47] D. Viladot, M. Véron, M. Gemmi, F. Peiró, J. Portillo, S. Estradé, J. Mendoza, N.

Llorca-Isern, S. Nicolopoulos, Orientation and phase mapping in the transmission electron microscope using precession-assisted diffraction spot recognition: state-of-the-art results, J. Microsc., 252 (2013) 23-34.

[48] M.H. Loretto, R.E. Smallman, Defect analysis in electron microscopy, (1975).

[49] D.J. Dingley, Orientation imaging microscopy for the transmission electron microscope, Microchim. Acta, 155 (2006) 19-29.

[50] D. Barbier, N. Gey, N. Bozzolo, S. Allain, M. Humbert, EBSD for analysing the twinning microstructure in fine-grained TWIP steels and its influence on work hardening, J. Microsc., 235 (2009) 67-78.

[51] S. Zaefferer, New developments of computer-aided crystallographic analysis in transmission electron microscopy, J Appl Crystallogr, 33 (2000) 10-25.

[52] E.F. Rauch, J. Portillo, S. Nicolopoulos, D. Bultreys, S. Rouvimov, P. Moeck, Automated nanocrystal orientation and phase mapping in the transmission electron microscope on the basis of precession electron diffraction, Z Kristallogr, 225 (2010) 103-109.

[53] T. White, A. Eggeman, P. Midgley, Is precession electron diffraction kinematical? Part I::" Phase-scrambling" multislice simulations, Ultramicroscopy, 110 (2010) 763-770.

[54] E.F. Rauch, L. Dupuy, Comments on 'On the reliability of fully automatic indexing of electron diffraction patterns obtained in a transmission electron microscope' by Morawiec \& Bouzy (2006), J Appl Crystallogr, 39 (2006) 104-105.

[55] A.J. Wilkinson, G. Meaden, D.J. Dingley, High-resolution elastic strain measurement from electron backscatter diffraction patterns: new levels of sensitivity, Ultramicroscopy, 106 (2006) 307-313.

[56] T. Britton, J. Jiang, R. Clough, E. Tarleton, A. Kirkland, A. Wilkinson, Assessing the precision of strain measurements using electron backscatter diffraction-part 1: Detector assessment, Ultramicroscopy, 135 (2013) 126-135.

[57] T. Britton, J. Jiang, R. Clough, E. Tarleton, A. Kirkland, A. Wilkinson, Assessing the precision of strain measurements using electron backscatter diffraction-Part 2: Experimental demonstration, Ultramicroscopy, 135 (2013) 136-141.

[58] E.F. Rauch, M. Véron, Automated crystal orientation and phase mapping in TEM, Materials Characterization, 98 (2014) 1-9.

[59] I. Ghamarian, Y. Liu, P. Samimi, P.C. Collins, Development and application of a novel precession electron diffraction technique to quantify and map deformation structures in highly deformed materials - as applied to ultrafine-grained titanium, Acta Mater., 79 (2014) 203-215. [60] M.A. Meyers, A. Mishra, D.J. Benson, Mechanical properties of nanocrystalline materials, Prog Mater Sci, 51 (2006) 427-556.

[61] R.A. Schwarzer, J. Sukkau, Automated crystal orientation mapping (ACOM) with a computer-controlled TEM by interpreting transmission Kikuchi patterns, Mater. Sci. Forum, Trans Tech Publ, 1998, pp. 215-222.

[62] Y.M. Wang, F. Sansoz, T. LaGrange, R.T. Ott, J. Marian, T.W. Barbee Jr, A.V. Hamza, Defective twin boundaries in nanotwinned metals, Nat. Mater., 12 (2013) 697-702.

[63] L. Cao, K. Ganesh, L. Zhang, O. Aubel, C. Hennesthal, M. Hauschildt, P.J. Ferreira, P.S. Ho, Grain structure analysis and effect on electromigration reliability in nanoscale $\mathrm{Cu}$ interconnects, Appl Phys Lett, 102 (2013) 131907. 
[64] A. Arsenlis, D. Parks, Crystallographic aspects of geometrically-necessary and statisticallystored dislocation density, Acta Mater., 47 (1999) 1597-1611.

[65] W. Pantleon, Resolving the geometrically necessary dislocation content by conventional electron backscattering diffraction, Scripta Mater., 58 (2008) 994-997.

[66] T. Benjamin Britton, A.J. Wilkinson, Stress fields and geometrically necessary dislocation density distributions near the head of a blocked slip band, Acta Mater., 60 (2012) 5773-5782.

[67] T. Britton, H. Liang, F. Dunne, A. Wilkinson, The effect of crystal orientation on the indentation response of commercially pure titanium: experiments and simulations, Proceedings of the Royal Society A: Mathematical, Physical and Engineering Science, 466 (2010) 695-719. [68] J. Jiang, T. Britton, A. Wilkinson, Evolution of dislocation density distributions in copper during tensile deformation, Acta Mater., 61 (2013) 7227-7239.

[69] P. Littlewood, A. Wilkinson, Geometrically necessary dislocation density distributions in cyclically deformed Ti-6Al-4V, Acta Mater., 60 (2012) 5516-5525.

[70] P. Littlewood, T. Britton, A. Wilkinson, Geometrically necessary dislocation density distributions in Ti-6Al-4V deformed in tension, Acta Mater., 59 (2011) 6489-6500.

[71] S. Sun, B. Adams, W. King, Observations of lattice curvature near the interface of a deformed aluminium bicrystal, Philosophical Magazine A, 80 (2000) 9-25.

[72] T. Ruggles, D. Fullwood, Estimations of Bulk Geometrically Necessary Dislocation Density Using High Resolution EBSD, Ultramicroscopy, 133 (2013) 8-15.

[73] D. Field, P. Trivedi, S. Wright, M. Kumar, Analysis of local orientation gradients in deformed single crystals, Ultramicroscopy, 103 (2005) 33-39.

[74] A.J. Wilkinson, D. Randman, Determination of elastic strain fields and geometrically necessary dislocation distributions near nanoindents using electron back scatter diffraction, Philos Mag, 90 (2010) 1159-1177.

[75] S.S. Rao, S. Rao, Engineering optimization: theory and practice, John Wiley \& Sons, 2009.

[76] W. Pantleon, W. He, T. Johansson, C. Gundlach, Orientation inhomogeneities within individual grains in cold-rolled aluminium resolved by electron backscatter diffraction, Mater.

Sci. Eng. A, 483 (2008) 668-671.

[77] F. Bachmann, R. Hielscher, H. Schaeben, Texture analysis with MTEX-free and open source software toolbox, Solid State Phenomena, 160 (2010) 63-68.

[78] X.Y. Zhang, B. Li, X.L. Wu, Y.T. Zhu, Q. Ma, Q. Liu, P.T. Wang, M.F. Horstemeyer, Twin boundaries showing very large deviations from the twinning plane, Scripta Mater., 67 (2012) 862-865.

[79] V. Randle, A methodology for grain boundary plane assessment by single-section trace analysis, Scripta Mater., 44 (2001) 2789-2794.

[80] G.S. Rohrer, D.M. Saylor, B. El-Dasher, B.L. Adams, A.D. Rollett, P. Wynblatt, The distribution of internal interfaces in polycrystals, Z. Metallkd, 95 (2004) 197-214.

[81] H. Beladi, G.S. Rohrer, A.D. Rollett, V. Tari, P.D. Hodgson, The distribution of intervariant crystallographic planes in a lath martensite using five macroscopic parameters, Acta Mater., 63 (2014) 86-98.

[82] L.S. Toth, C.F. Gu, Modeling of disorientation axis distribution in severely deformed copper, Scripta Mater., 69 (2013) 183-186.

[83] L.S. Tóth, Y. Estrin, R. Lapovok, C. Gu, A model of grain fragmentation based on lattice curvature, Acta Mater., 58 (2010) 1782-1794. 
[84] L.S. Tóth, B. Beausir, C.F. Gu, Y. Estrin, N. Scheerbaum, C.H.J. Davies, Effect of grain refinement by severe plastic deformation on the next-neighbor misorientation distribution, Acta Mater., 58 (2010) 6706-6716.

[85] B. Beausir, C. Fressengeas, N.P. Gurao, L.S. Tóth, S. Suwas, Spatial correlation in grain misorientation distribution, Acta Mater., 57 (2009) 5382-5395.

[86] G. Viswanathan, E. Lee, D.M. Maher, S. Banerjee, H.L. Fraser, Direct observations and analyses of dislocation substructures in the $\alpha$ phase of an $\alpha / \beta$ Ti-alloy formed by nanoindentation, Acta Mater., 53 (2005) 5101-5115.

[87] A. Ma, F. Roters, D. Raabe, A dislocation density based constitutive model for crystal plasticity FEM including geometrically necessary dislocations, Acta Mater., 54 (2006) 21692179.

[88] A. Ma, F. Roters, D. Raabe, On the consideration of interactions between dislocations and grain boundaries in crystal plasticity finite element modeling - Theory, experiments, and simulations, Acta Mater., 54 (2006) 2181-2194.

[89] F. Roters, P. Eisenlohr, L. Hantcherli, D.D. Tjahjanto, T.R. Bieler, D. Raabe, Overview of constitutive laws, kinematics, homogenization and multiscale methods in crystal plasticity finiteelement modeling: Theory, experiments, applications, Acta Mater., 58 (2010) 1152-1211. 


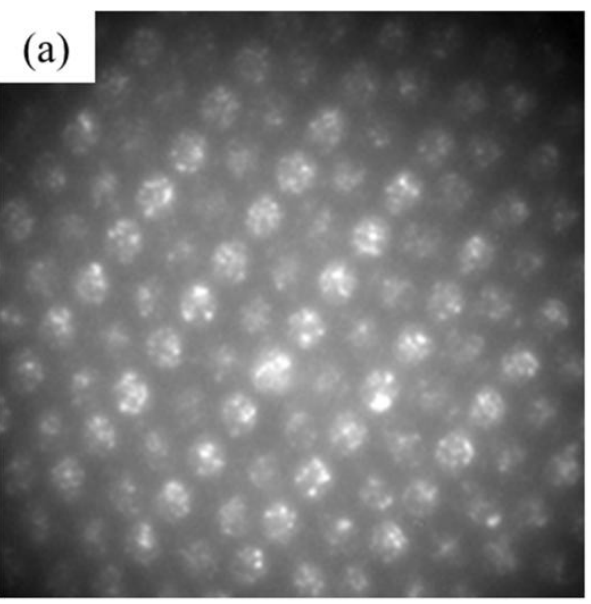

(b)
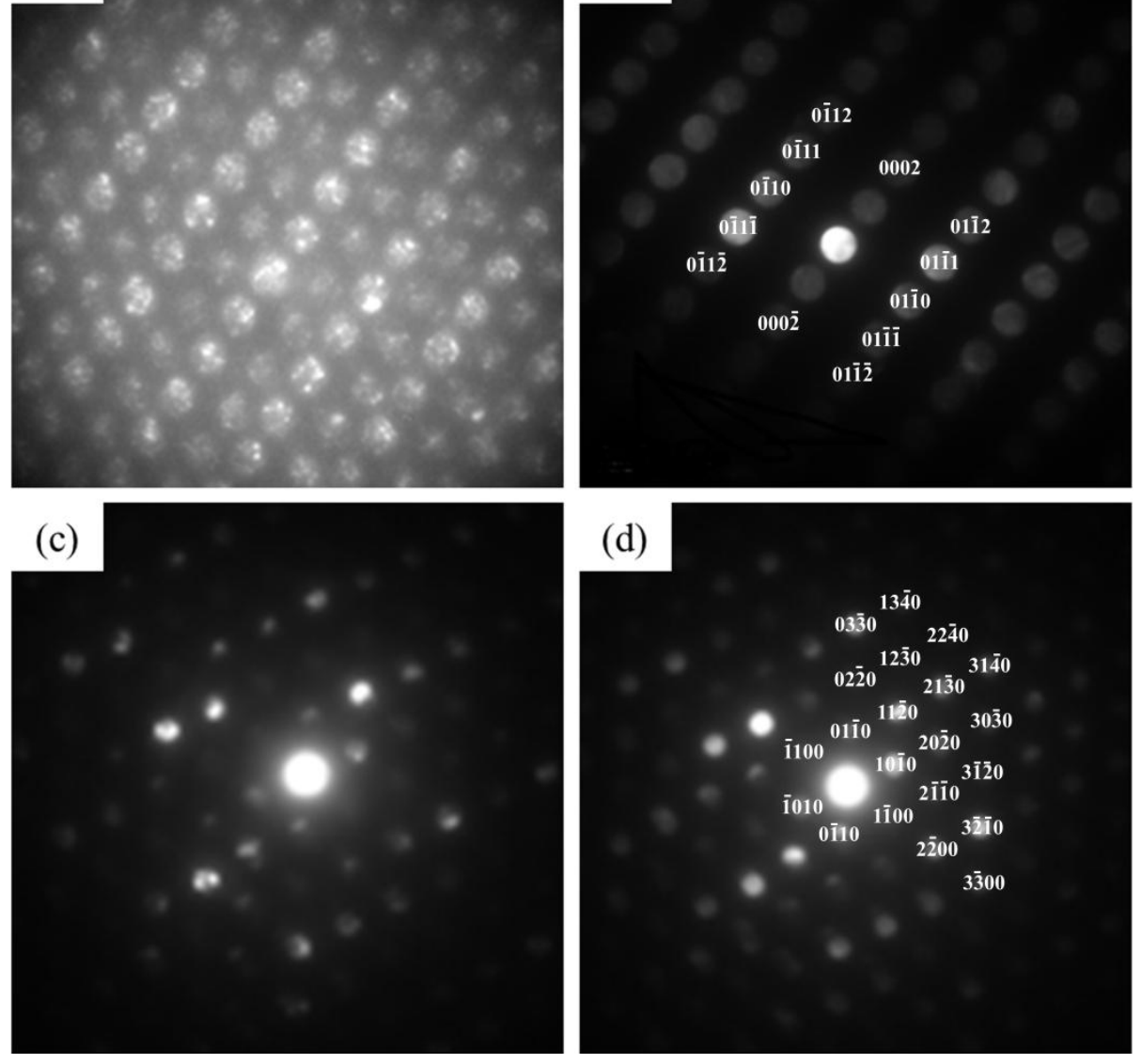

(d)

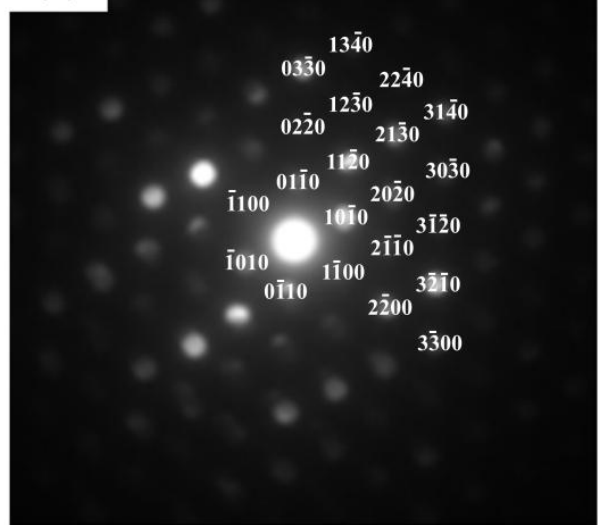

Figure 1. 


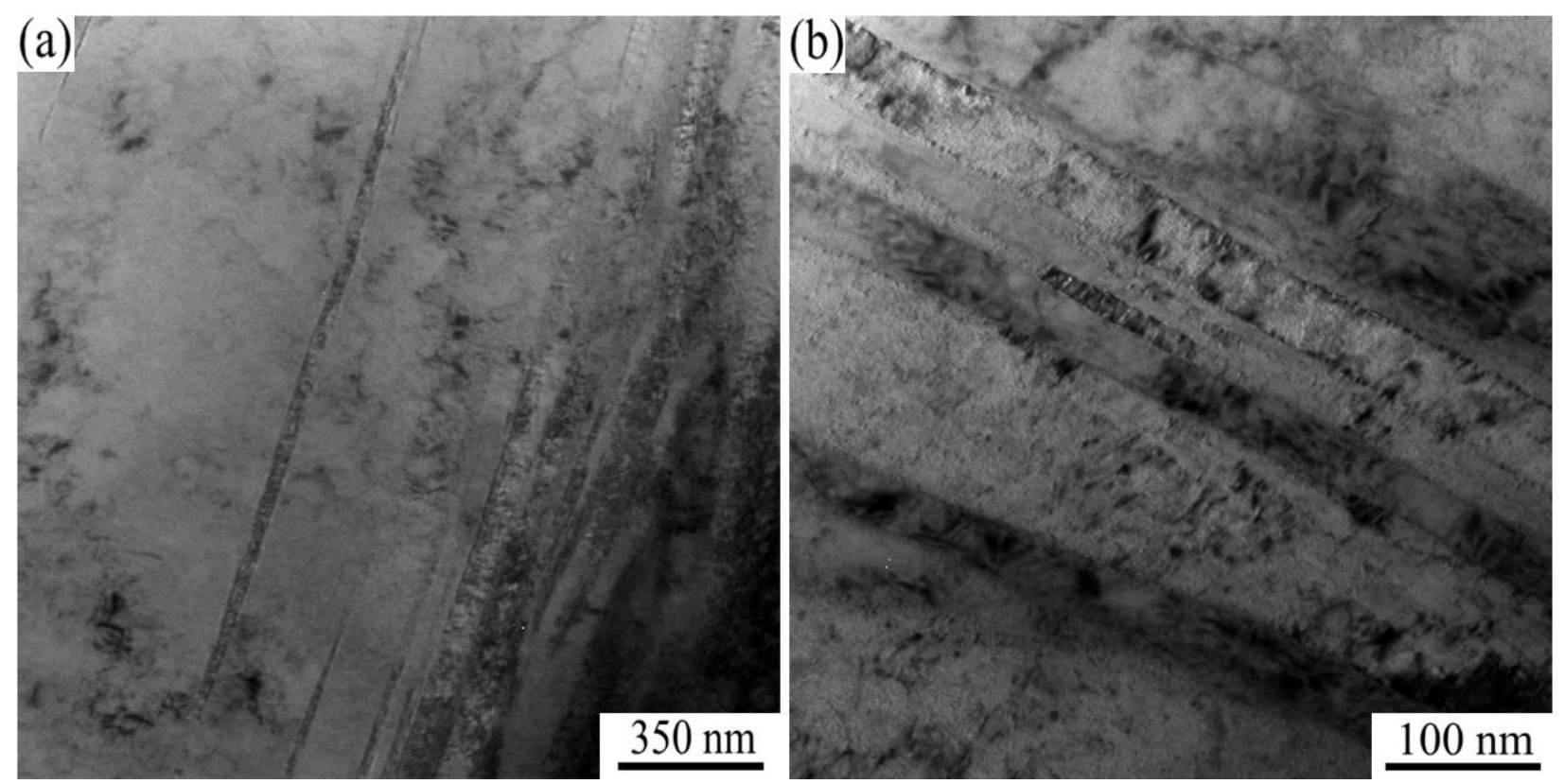

Figure 2. 


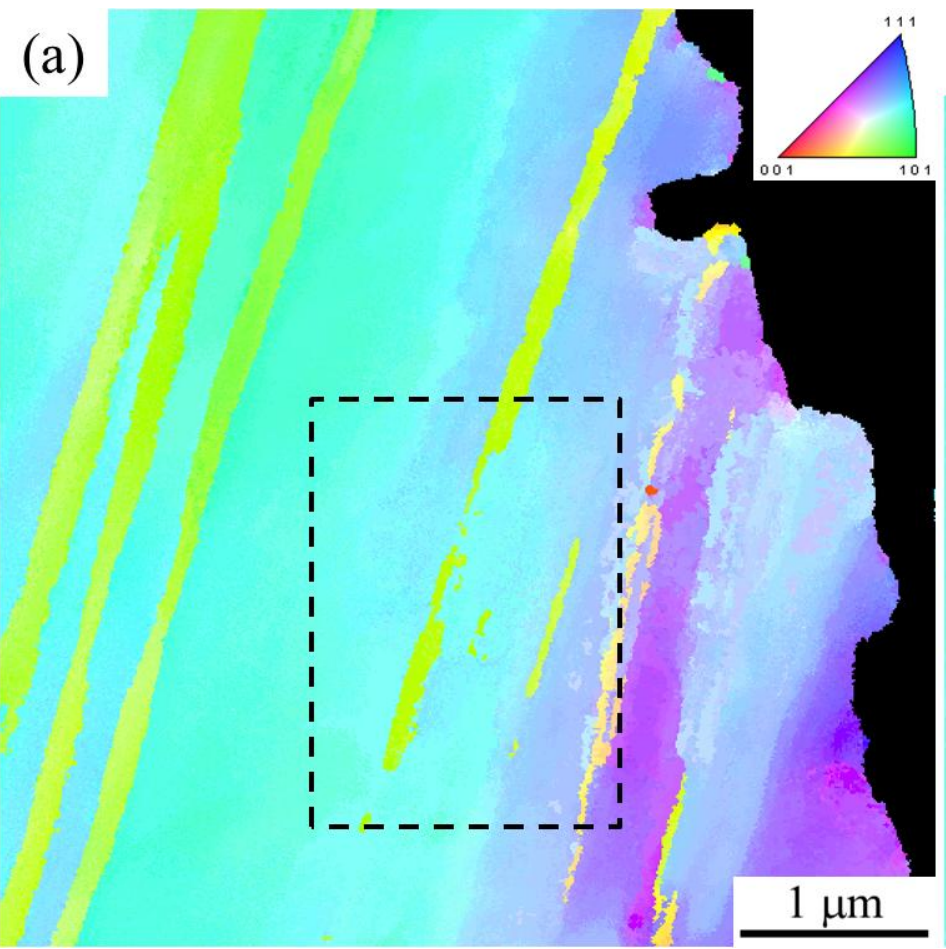

(b)

Figure 3. 

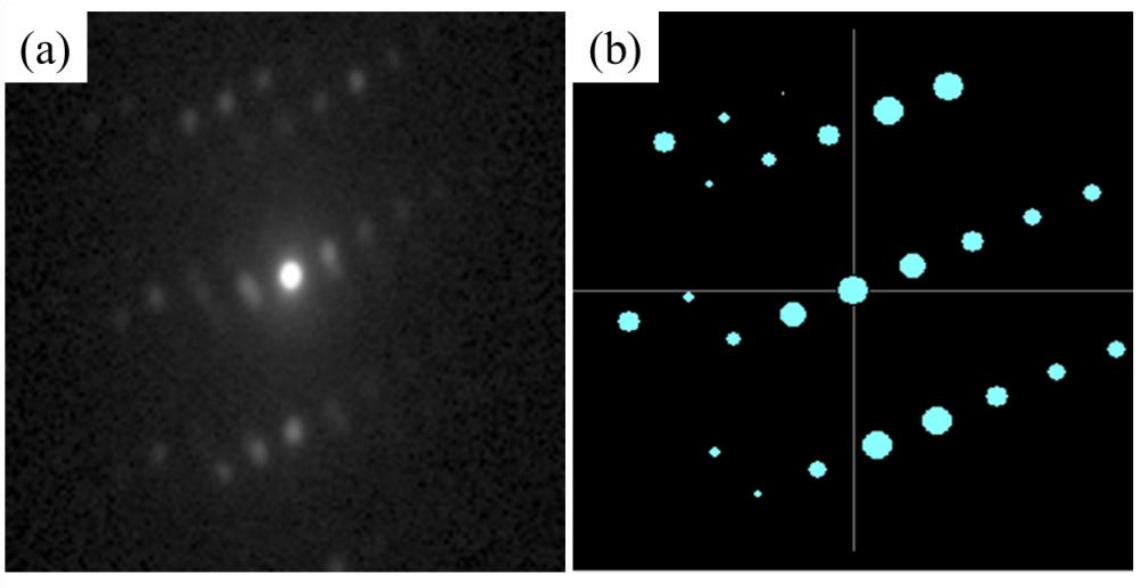

(c)

\section{(e)}

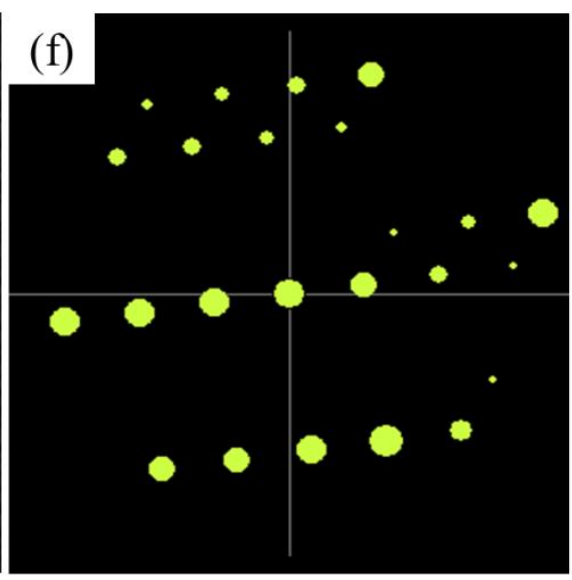

(g)

(d)
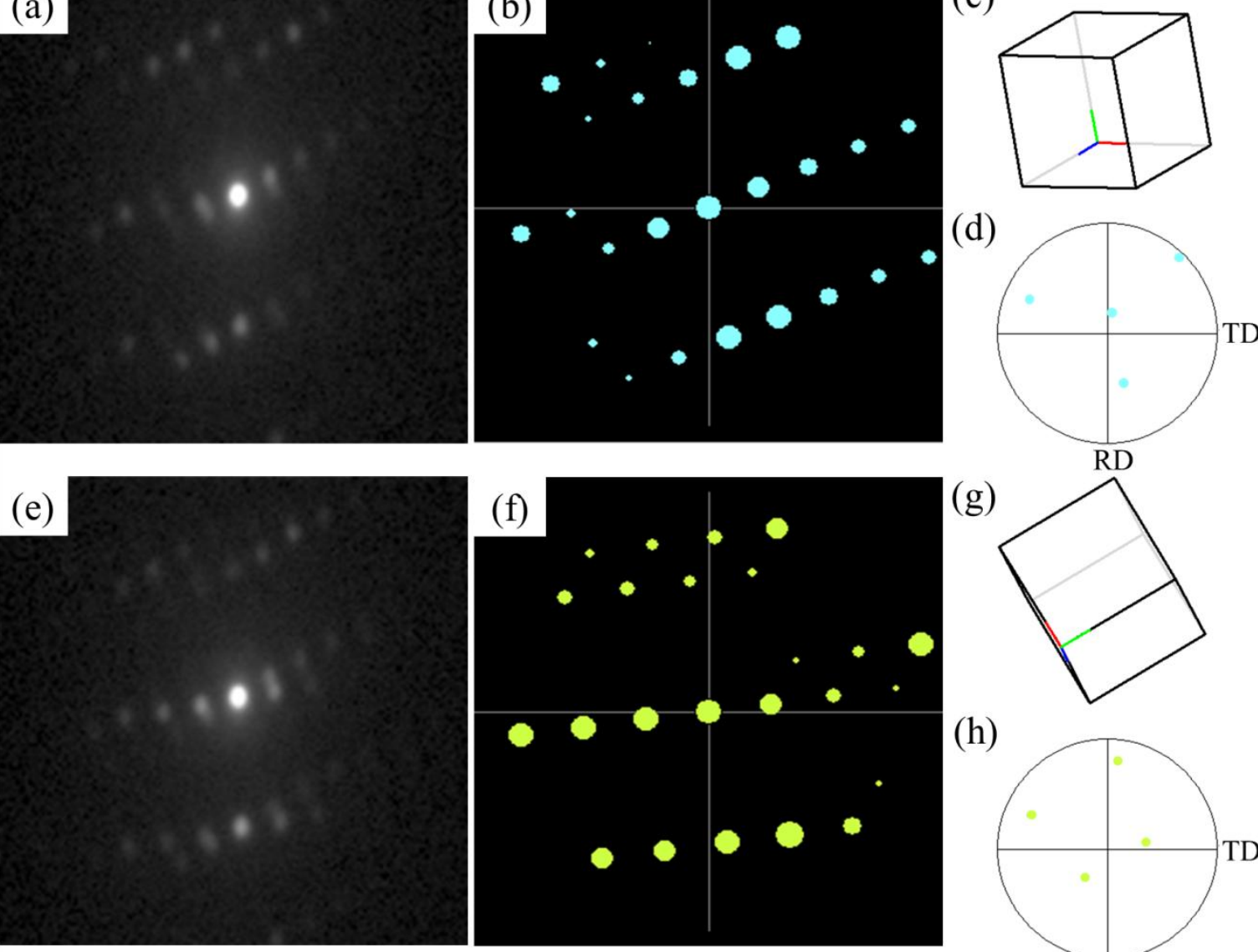

(k)

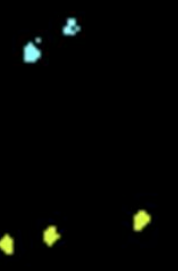

-

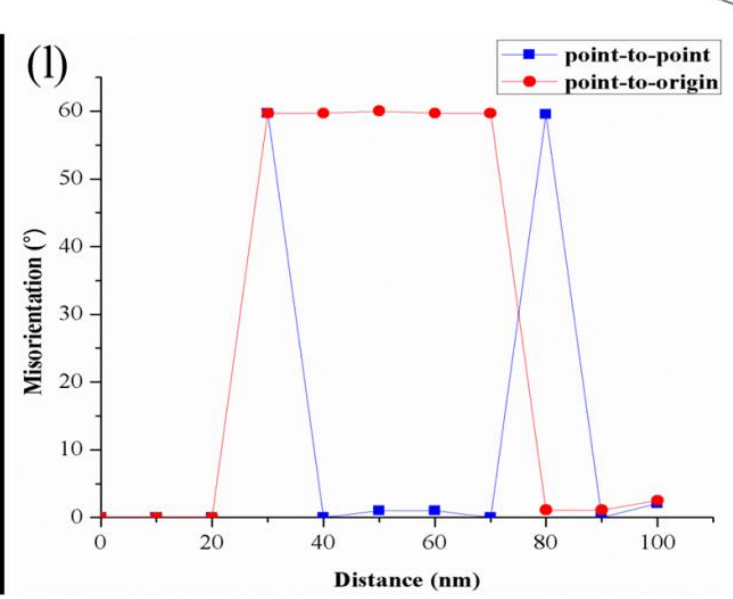

Figure 4 . 

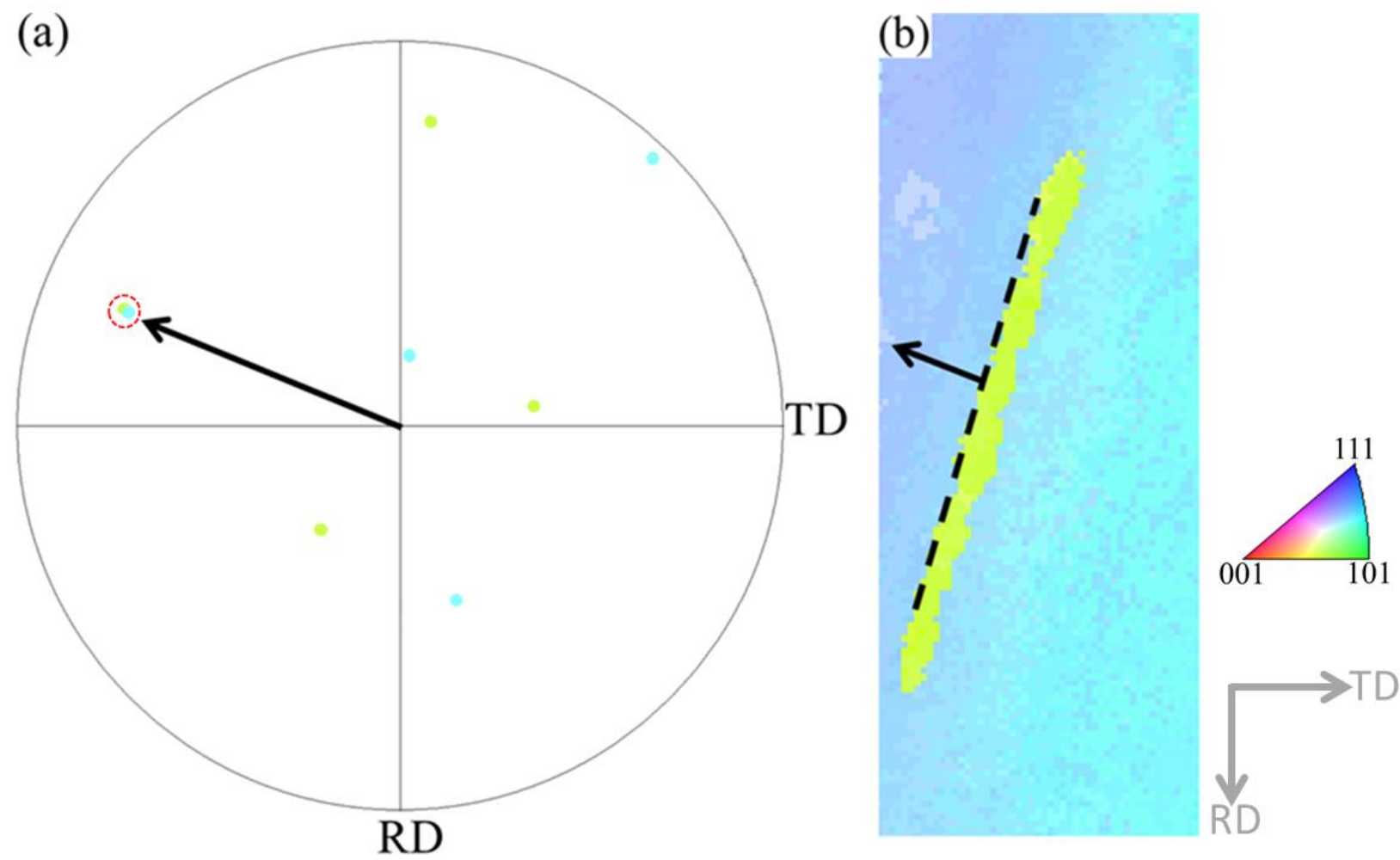

Figure 5. 

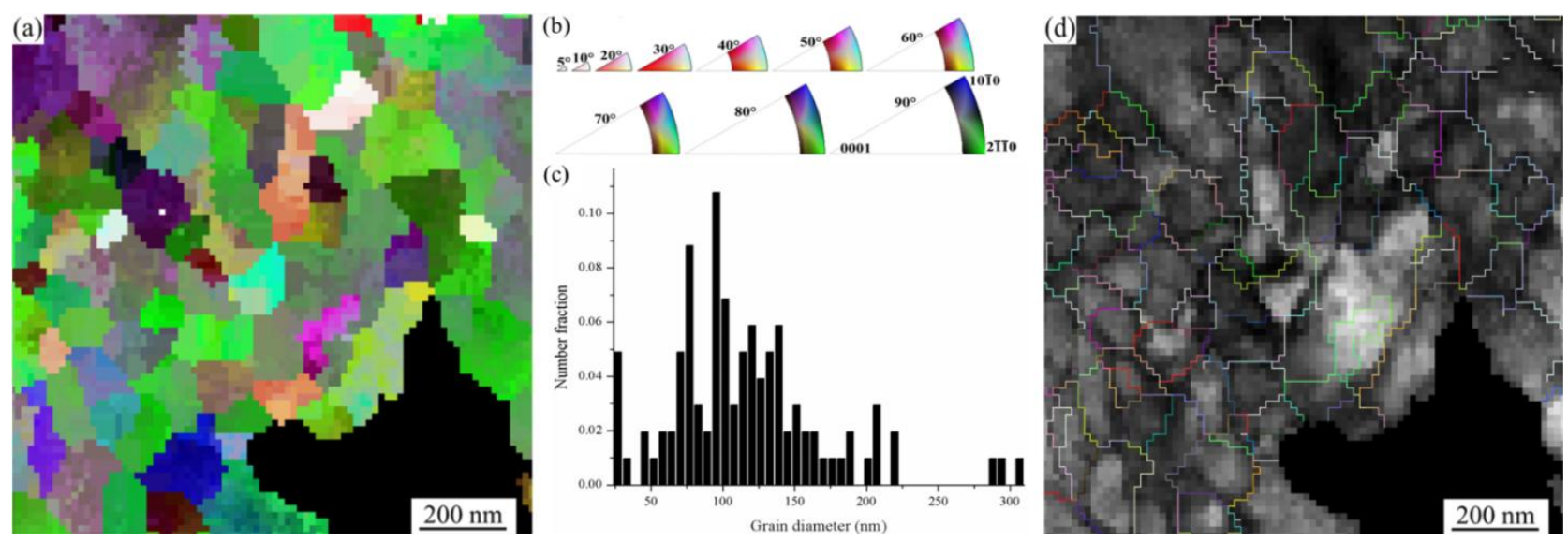

Figure 6. 


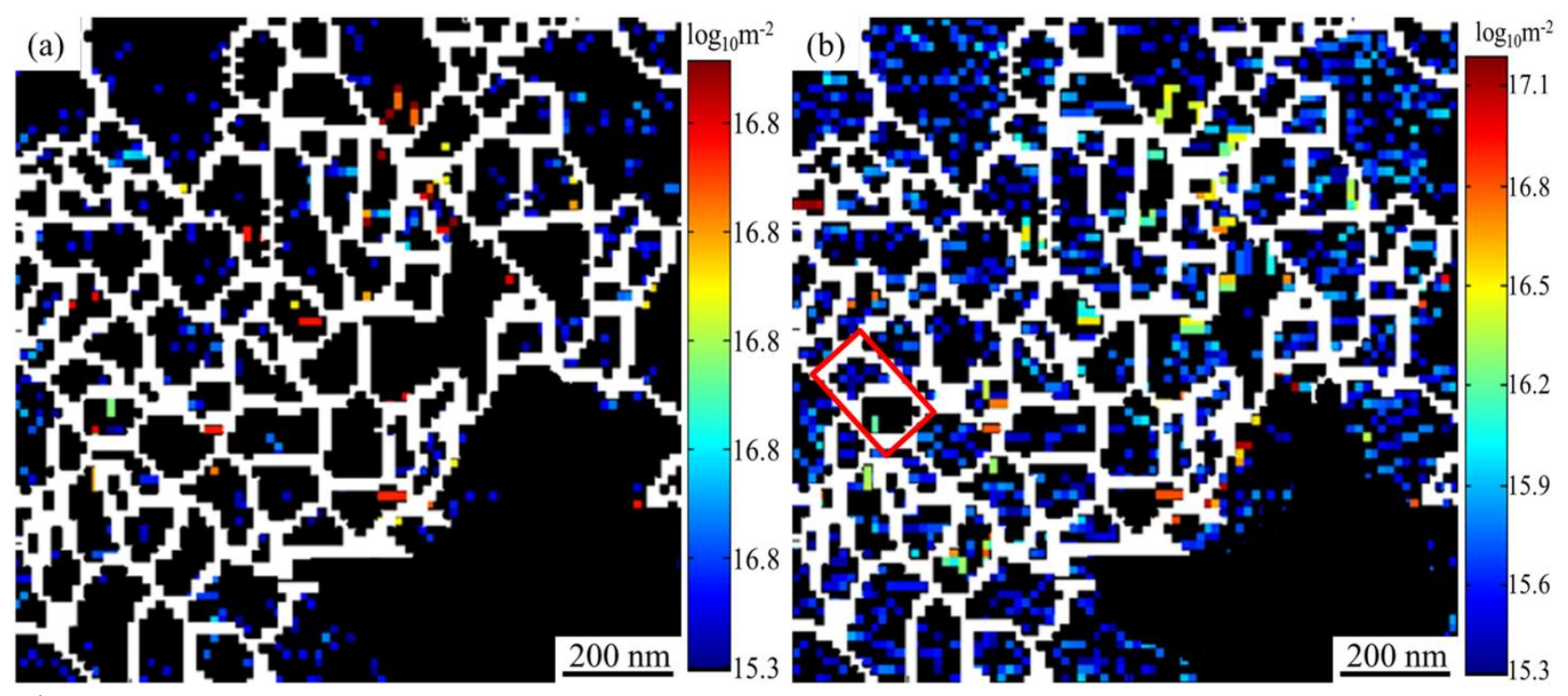

Figure 7. 
Figure 1. Contribution of precessing the direct beam on sharpening the acquired diffraction pattern iamges (a) The diffraction pattern of a crystal oriented close to $2 \overline{1} \overline{1} 0$ zone axis when the direct beam is not precessed ("PED off" condition) (b) The same situation as "a" with precessing the direct beam for $1.3^{\circ}$ ("PED on condition") (c) The diffraction pattern of a crystal oriented far from 0001 zone axis in a PED off condition (d) The same situation as "c" with PED on condition.

Figure 2. Many-beam bright field TEM images of a severely deformed nickle alloy (a) Samedirection narrow bands (b) Bands with less than $100 \mathrm{~nm}$ width

Figure 3. PED recorded orientation dataset for a severely deformed nickel sample (a) Plotted IPF map along normal direction (ND) (b) Enlarge view of the region surrounded by dashed rectangle in "a"

Figure 4.Characterization of the nanotwin exists between $A$ and $B$ points in Fig. 3b (a) Recorded diffraction pattern from an area out of the nanotwin (b) Simulated diffraction pattern which mostly matches the diffraction pattern shown in "a" (c) Schematic of the crystal orientation (d) 111 pole figure (e) Acquired diffraction pattern from a region inside the nanotwin (f) Most mathced simulated diffraction pattern for the one shown in "e" (g) Schematic of the crystal orientation (h) 111 pole figure (k) Differences between "a" and "e" diffraction patterns (l) Pointto-point and point-to-origin misorientations along the hypothetical $A B$ line.

Figure 5. Coherency analysis via trace method of a detected nanotwin boundary (a) 111 pole figure of the matrix and nanotwin shown in "b" (The solid vector represents the twin boundary trace normal while the dot arrow shows the oirentation of the boundary trace normal in the fully coherent condition) (b) IPF map along normal direction (ND). The norml direction to the reconstructed $\Sigma 3$ nanotwin boundary $\left(60^{\circ}<111>\right)$ is drawn. 
Figure 6. Characterization of a severely deformed commercially pure titanium alloy (a) Quaternion misorientation plot (b) The color legend of quaternion misorientation plot (c) Grain size diameter distribution (d) Grain boundary in quaternion color overlaid on the index map

Figure 7. Dislocation density distribution plots in a commercially pure titanium alloy (a) $\langle\boldsymbol{a}\rangle$ type screw dislocation with $b=0.3[\overline{2} 110]$ (b) $\langle\boldsymbol{c}+\boldsymbol{a}\rangle$ pyramidal type edge dislocation with $b=0.3[\overline{2} 11 \overline{3}]$ 